\title{
Editorial
}

\section{Recent Advances on Methods and Applications of Nonlinear Differential Equations}

\author{
Chaudry Masood Khalique, ${ }^{1}$ Mufid Abudiab, ${ }^{2}$ Fazal Mahmood Mahomed, \\ Hossein Jafari, ${ }^{4}$ and Guo-Cheng $\mathrm{Wu}^{5}$ \\ ${ }^{1}$ International Institute for Symmetry Analysis and Mathematical Modelling, Department of Mathematical Sciences, \\ North-West University, Mafikeng Campus, Private Bag X2046, Mmabatho 2735, South Africa \\ ${ }^{2}$ Department of Mathematics and Statistics, Texas A\&M University-Corpus Christi, 6300 Ocean Drive, \\ Corpus Christi, TX 78412, USA \\ ${ }^{3}$ Centre for Differential Equations, Continuum Mechanics and Applications, School of Computational and Applied Mathematics, \\ University of the Witwatersrand, Johannesburg 2050, South Africa \\ ${ }^{4}$ Department of Mathematics, Faculty of Mathematical Sciences, University of Mazandaran, Babolsar, Iran \\ ${ }^{5}$ Department of Mathematics and Information Science, Neijiang Normal University, Neijiang 641112, China
}

Correspondence should be addressed to Chaudry Masood Khalique; masood.khalique@nwu.ac.za

Received 31 January 2014; Accepted 31 January 2014; Published 11 March 2014

Copyright (C) 2014 Chaudry Masood Khalique et al. This is an open access article distributed under the Creative Commons Attribution License, which permits unrestricted use, distribution, and reproduction in any medium, provided the original work is properly cited.

Nonlinear differential equations have been extensively used to mathematically model many of the interesting and important phenomena that are observed in many areas of science and technology. They are inspired by problems which arise in diverse fields such as economics, biology, fluid dynamics, physics, differential geometry, engineering, control theory, materials science, and quantum mechanics.

The purpose of this special issue is to highlight some recent developments in methods and applications of nonlinear differential equations. The majority of the papers contained in this special issue are based on areas of research ranging from functional analytic techniques to Lie symmetry and singularity methods as well as numerical methods that are applied to both partial and ordinary differential equations. There are papers which deal with fractional and stochastic differential equations and in addition papers analysing equations that arise in engineering as well as classical and fluid mechanics and finance.

Hundred and fifteen papers were submitted for possible publication in this special issue. After a rigorous reviewing process, fifty-three papers were finally accepted for publication.
We very much hope that the papers published in this special issue will be useful to a large community of researchers and will arouse further research in the topics presented as well as in the connected fields.

\section{Acknowledgments}

We wish to express our sincere thanks to all the authors who contributed to this Special Issue and to its success. We take this opportunity to thank the reviewers who worked very hard and diligently to review all the submitted manuscripts and thus added to the high standards achieved.

\author{
Chaudry Masood Khalique \\ Mufid Abudiab \\ Fazal Mahmood Mahomed \\ Hossein Jafari \\ Guo-Cheng Wu
}




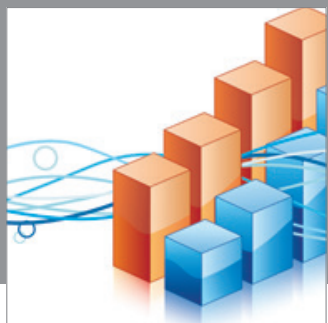

Advances in

Operations Research

mansans

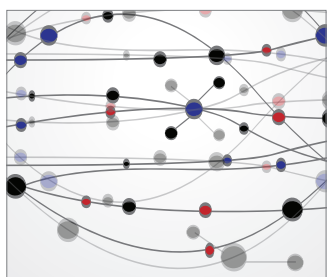

The Scientific World Journal
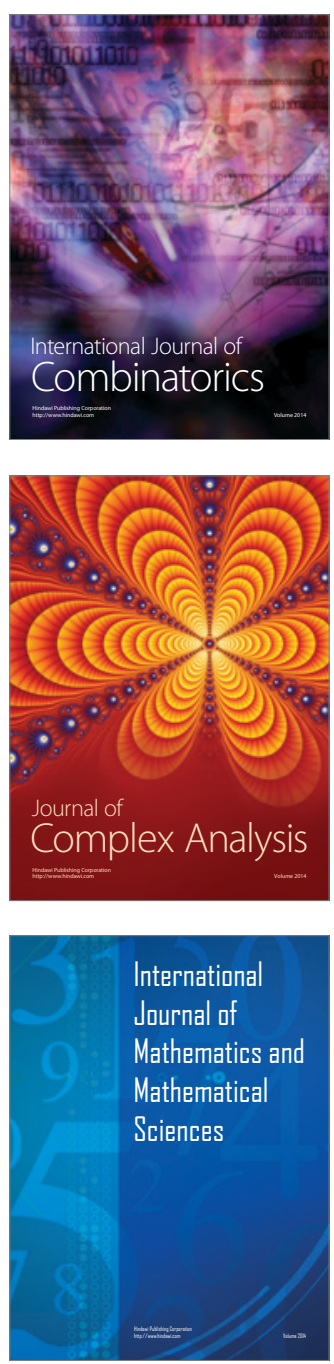
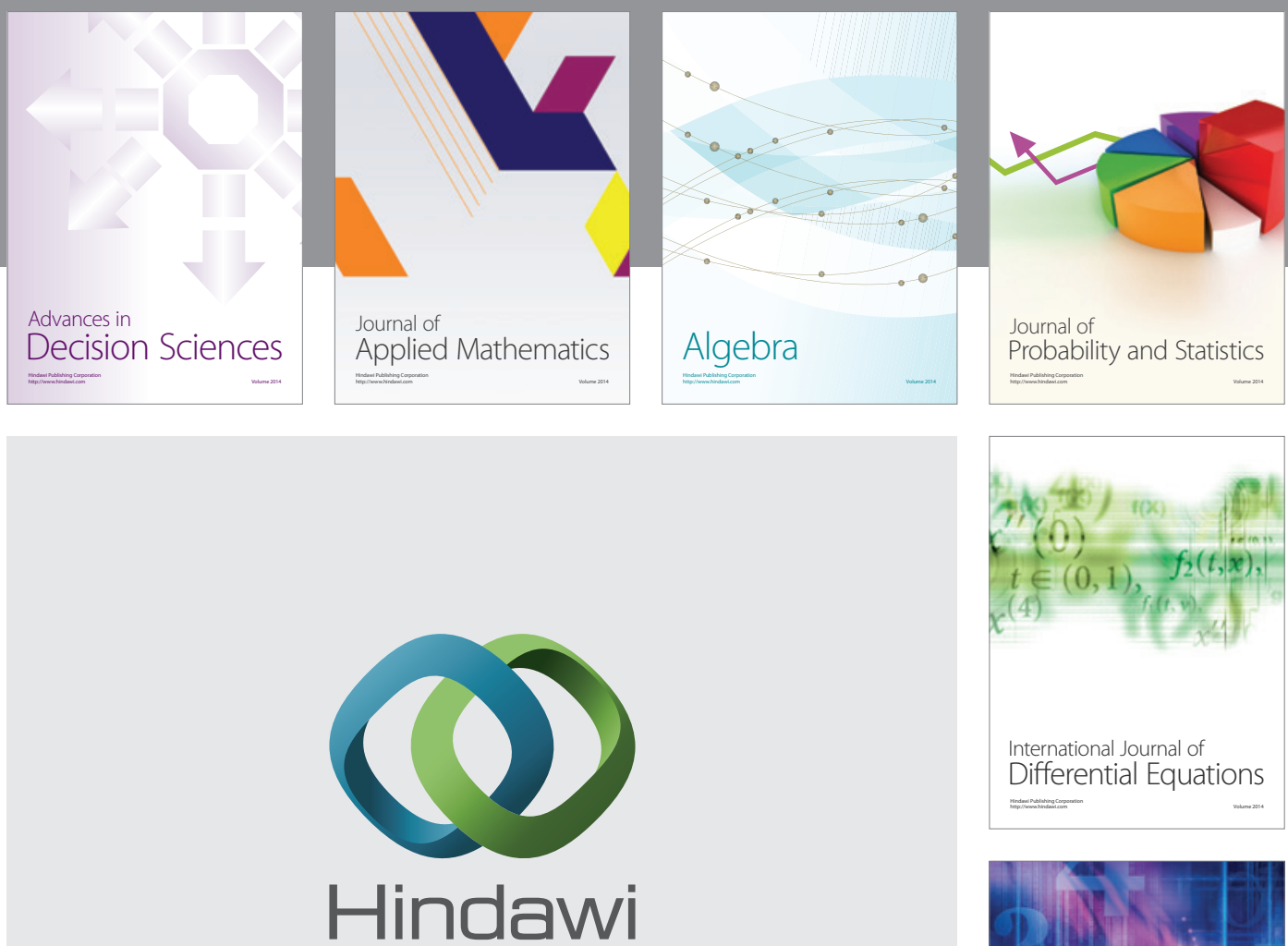

Submit your manuscripts at http://www.hindawi.com
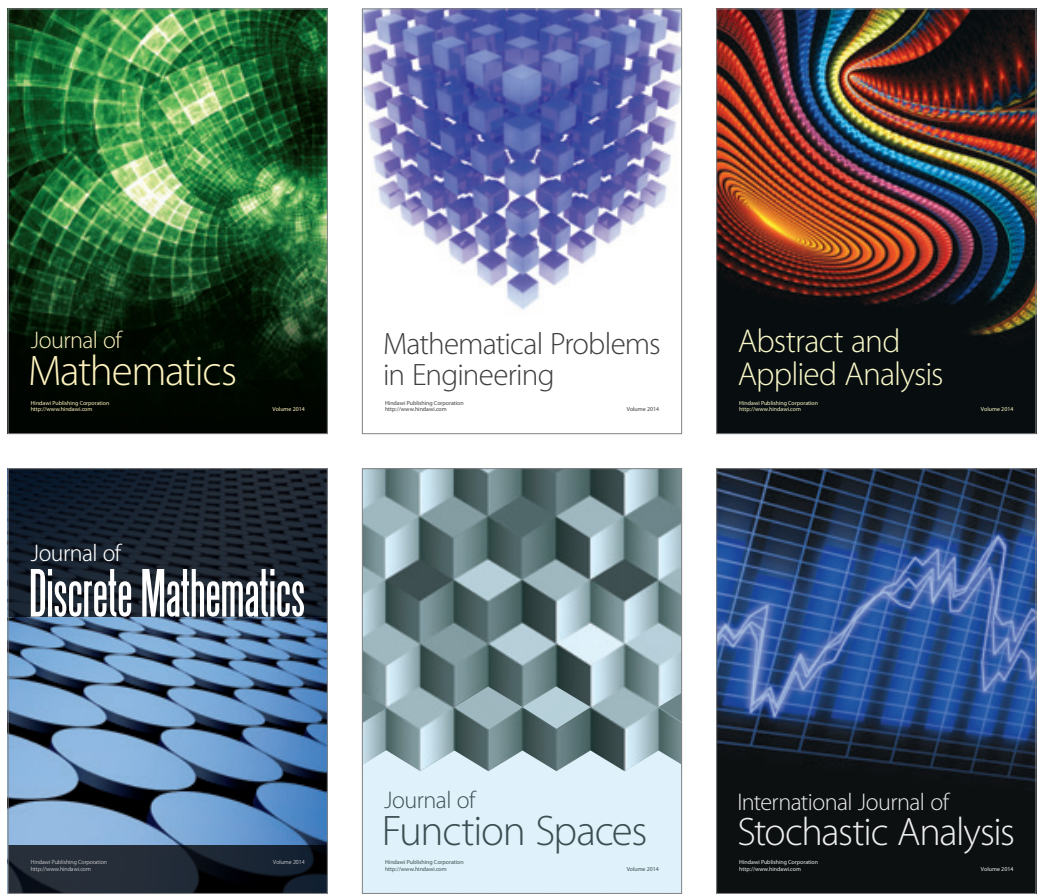

Journal of

Function Spaces

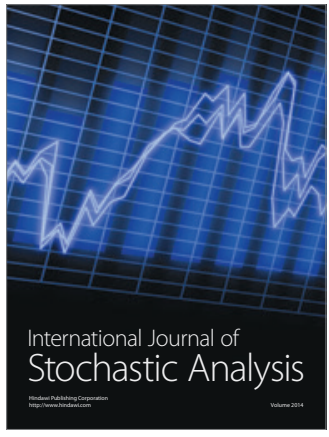

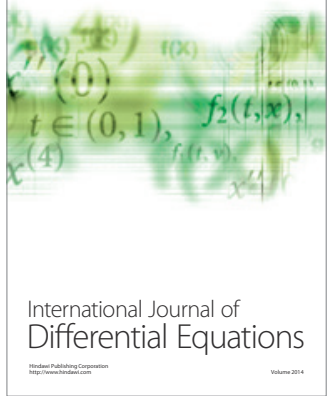
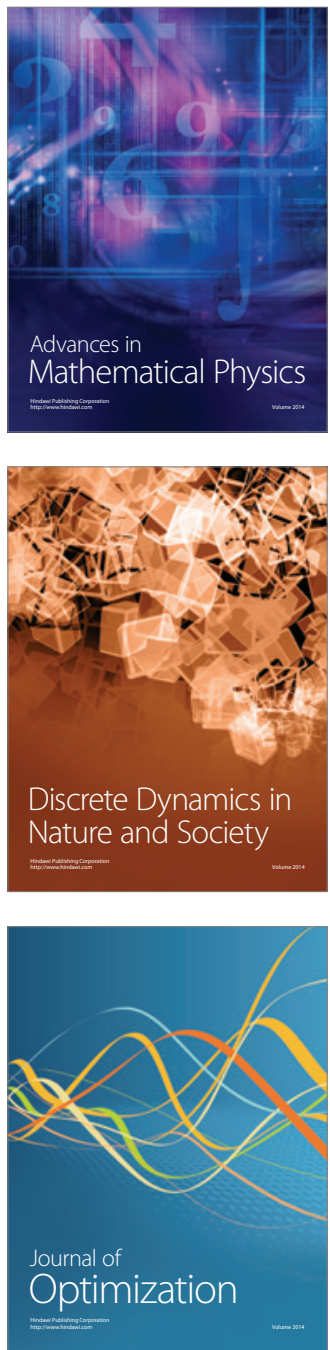